\title{
Accuracy Investigation of FDM, FEM and MoM for a Numerical Solution of the 2D Laplace's Differential Equation for Electrostatic Problems
}

\author{
Bojan Glushica ${ }^{1 *}$, Andrijana Kuhar ${ }^{1}$, and Vesna Arnautovski Toseva ${ }^{1}$ \\ ${ }^{1}$ Ss. Cyril and Methodius University in Skopje, Faculty of Electrical Engineering and Information \\ Technologies, Skopje, North Macedonia \\ *E-mail of corresponding author: glushica@feit.ukim.edu.mk
}

\begin{abstract}
Laplace's differential equation is one of the most important equations which describe the continuity of a system in various fields of engineering. As a system gets more complex, the need for solving this equation numerically rises. In this paper we present an accuracy investigation of three of the most significant numerical methods used in computational electromagnetics by applying them to solve Laplace's differential equation in a two-dimensional domain with Dirichlet boundary conditions. We investigate the influence of discretization on the relative error obtained by applying each method. We point out advantages and disadvantages of the investigated computational methods with emphasis on the hardware requirements for achieving certain accuracy.
\end{abstract}

Received 12 June 2021

Accepted 3 September 2021

Published 22 December 2021

Keywords: FDM, FEM, MoM, Laplace's equation, Dirichlet boundary conditions.

\section{Introduction}

The importance of numerical methods in engineering and science in general is a well-known fact in the contemporary time of available advanced hardware and software resources. In this paper we apply three of the most significant numerical methods used in computational electromagnetics, i.e. the finite difference method (FDM), the finite element method (FEM) and the method of moments (MoM) to solve Laplace's differential equation in a two-dimensional domain with Dirichlet boundary conditions. The goal is to investigate the accuracy of the numerical methods in terms of discretization and hardware requirements.

Laplace's equation is a differential equation of the second order that describes a number of electrical and physical phenomena in engineering and especially in the field of biomedicine. For example, in electrostatic problems it is used to describe a phenomenon of crucial importance: the behaviour of the electric scalar potential and its gradient.

Previously, a comparison of various methods has been made in [1]. Many of those methods and their variations are still used. However, the comparison made is only mathematical and no results are provided. The general Helmholtz equation has been solved using MoM in [2] and the obtained results are compared with the results from FDM and FEM. Furthermore, the boundary conditions for the problem are of Dirichlet and Neumann mixed type, which are only used for specific cases. A direct comparison of values obtained by applying FDM,

(C) The Author(s) 2021. Published by CIEES Association Press. This work is licensed under a Creative Commons Attribution-NonCommercialNoDerivs 4.0 Generic License.
FEM and MoM for solving Laplace's equation on the same system has not yet been published, to the best of the authors' knowledge.

The formulation of the system is presented in second section. In section 3 of this paper we give an overview of the implemented methods and then investigate the accuracy of the obtained results for the Laplace's equation by these methods respectively on a $2 \mathrm{D}$ rectangular problem with various values for the Dirichlet boundary condition. Verification of the calculations is performed by comparison with the analytical solution for simple cases and presented in the results and discussion section (section 4). In section 5 conclusions of the accuracy investigation are summarized.

\section{Formulation of the problem}

We shall first define a suitable problem in order to properly investigate the accuracy of the methods for a $2 \mathrm{D}$ system. Based on the implementations of the methods which follow in the next section, we will consider a rectangular domain $R$ defined by a contour $C$ in Cartesian coordinate system. The boundary conditions on the contour $C$ can be defined by

$\alpha \varphi+\beta \frac{\partial \varphi}{\partial n}=\gamma$

where $\alpha, \beta, \gamma$ are known functions on $C, \varphi$ is the potential and $\partial \varphi / \partial n$ is its normal derivative. We will consider only the Dirichlet boundary condition where only $\varphi$ is specified on $C$, i.e. $\alpha=1$ and $\beta=0$. The boundary values will be used to determine the potential distribution in the domain $R$. The discretisation of $C$ allows $R$ to be divided into equal rectangular elements. The methods were implemented for solving the Laplace's equation for this problem. 


\section{Overview of the implemented methods}

In this section the implementation of the three numerical methods (FDM, FEM and MoM) is described mathematically, pointing out their most important characteristics. The mathematical approach for obtaining the analytical solution is also discussed.

\subsection{Finite Difference Method}

The domain $R$ must be specified as a grid of $N \times$ $M$ nodal points. Let the distance between each point be $\Delta x$ and $\Delta y$ on the $x$ and $y$ axis respectively. This allows derivatives to be approximated with finite difference equations, detailed in [3]. The Laplace's equation expressed through the second order central difference approximation and the cardinal neighbours of a nodal point $(i, j)$ can be written as

$\frac{u_{i-1, j}-2 u_{i, j}+u_{i+1, j}}{\Delta x^{2}}+\frac{u_{i, j-1}-2 u_{i, j}+u_{i, j+1}}{\Delta y^{2}}=0$

where $u_{i, j}$ is the potential at point $(i, j)$ for $i, j \in \mathbb{N}, i<N$ and $j<M$. Generally $\Delta x$ and $\Delta y$ are not equal. With this in mind, solving for $u_{i, j}$ and rearranging (2) we get

$u_{i, j}{ }^{(k+1)}=\frac{\left[u_{i-1, j}+u_{i+1, j}\right]^{(k)}+\beta^{2}\left[u_{i, j-1}+u_{i, j+1}\right]^{(k)}}{2\left(1+\beta^{2}\right)}$

where $\beta=\Delta x / \Delta y, k=0,1,2, \ldots$ is the current iteration and $u_{i, j}{ }^{(k)}$ is the calculated potential after $k$ iterations. Because only the cardinal neighbours are taken into account in the calculation, the nodal points of the corners of the rectangular domain $R$ are ignored. Using (3) we can calculate the potential at each nodal point with arbitrary spacing $\Delta x$ and $\Delta y$, and an arbitrary grid size $N \times M$. Lastly, because of the iterative approach of the calculations, a maximum number of iterations or a minimal difference value between consecutive calculations on a same node need to be specified.

\subsection{Finite Element Method}

While FDM utilizes a differential equation approximation, FEM uses a weak formulation of the differential equation where the continuity requirements are preserved. By introducing an arbitrary weight function and integrating over a subdomain within the domain $R$, the weak formulation can be implemented [4]. The subdomain for our problem will be represented by a rectangular element described by 4 nodes. The solution domain $R$ is then divided into $N \times M$ number of elements. Each element and node are indexed. In matrix notation, the equation used for finding the potentials at each node $u_{i}$ is of the form

$[\mathrm{K}][\mathrm{U}]=[\mathrm{F}]$

where $[\mathrm{K}]$ is a $\mathrm{N} \cdot \mathrm{M}$ by $\mathrm{N} \cdot \mathrm{M}$ square matrix called the global stiffness matrix, $[\mathrm{U}]$ is a column matrix which contains the unknown values of each node $u_{i}$ and $[F]$ is also a column matrix containing the source and flux terms which are set to 0 for the Laplace's equation. In order to find the unknown values of $[\mathrm{U}],[\mathrm{K}]$ must be defined based on the used rectangular element. Let the distance between each node be $\mathrm{a}$ and $\mathrm{b}$ on the $\mathrm{x}$ and $\mathrm{y}$ axis respectively. We will use these distances to calculate the terms of the element coefficient matrix $\left[\mathrm{K}^{\mathrm{e}}\right]$. This matrix is derived from the weak formulation of the differential equation and is used to obtain the terms of $[\mathrm{K}]$. For an arbitrary rectangular element, the terms of $\left[\mathrm{K}^{\mathrm{e}}\right]$ can be analytically calculated and have the form

$\left[\mathrm{K}^{\mathrm{e}}\right]=$

$\frac{1}{6 a b}\left[\begin{array}{cccc}2\left(a^{2}+b^{2}\right) & a^{2}-2 b^{2} & -\left(a^{2}+b^{2}\right) & b^{2}-2 a^{2} \\ a^{2}-2 b^{2} & 2\left(a^{2}+b^{2}\right) & b^{2}-2 a^{2} & -\left(a^{2}+b^{2}\right) \\ -\left(a^{2}+b^{2}\right) & b^{2}-2 a^{2} & 2\left(a^{2}+b^{2}\right) & a^{2}-2 b^{2} \\ b^{2}-2 a^{2} & -\left(a^{2}+b^{2}\right) & a^{2}-2 b^{2} & 2\left(a^{2}+b^{2}\right)\end{array}\right]$

Each term of $\left[\mathrm{K}^{\mathrm{e}}\right]$ represents the influence each node has on the other nodes of the same element. In order to obtain the terms of $[\mathrm{K}]$, the values of the local indices of $\left[\mathrm{K}^{\mathrm{e}}\right]$ for each element of the domain need to be mapped to the global indices in $[\mathrm{K}]$. The unknown potentials at each node $u_{i}$ can now be obtained by solving the linear system (4). Because each rectangular element is described by 4 nodes, the solution for each element $u^{\mathrm{e}}$ is computed with

$\mathrm{u}^{\mathrm{e}}(\mathrm{x}, \mathrm{y})=\sum_{\mathrm{i}=1}^{4} \mathrm{u}_{\mathrm{i}}^{\mathrm{e}} \psi_{\mathrm{i}}^{\mathrm{e}}(\mathrm{x}, \mathrm{y})$

where $\psi_{i}^{e}(x, y)$ are interpolation functions and $u_{i}^{e}$ are the values at each node of the same element. The interpolation functions are constructed from the Lagrange interpolation polynomials. For rectangular elements this is a bilinear interpolation where the coordinate of interest is in the middle of the element at equal distance from each node, and is given by

$\mathrm{u}^{\mathrm{e}}(\mathrm{x}, \mathrm{y})=\frac{\mathrm{u}_{1}^{\mathrm{e}}+\mathrm{u}_{2}^{\mathrm{e}}+\mathrm{u}_{3}^{\mathrm{e}}+\mathrm{u}_{4}^{\mathrm{e}}}{4}$

The $[\mathrm{U}]$ matrix also contains the known values of the nodes positioned on the boundary. With this we can reduce the number of linear equations for the system (4). Furthermore, the values of the nodes at the corners of the domain $\mathrm{R}$ are undefined because at these nodes two different sides of the domain that can have different boundary values merge. This issue is resolved by averaging. It should be noted that although the $[\mathrm{K}]$ matrix is a sparse matrix, the accuracy of the solution depends directly on the number of elements in the domain. This causes the dimension of $[\mathrm{K}]$ to rise exponentially for a denser mesh which will increase the memory and computational requirements for the numerical solution.

\subsection{Method of Moments}

Integral equations are used to calculate the potential using the Method of Moments. The potential at an arbitrary point in R can be assumed that is caused by flux sources on $\mathrm{C}$ denoted by $\sigma(\mathrm{x}, \mathrm{y})$ [5]. For a $2 \mathrm{D}$ system the potential can be obtained by superposition in the form

$\varphi(x, y)=\oint_{C} \sigma\left(x^{\prime}, y^{\prime}\right) \ln \frac{K}{\sqrt{\left(x-x^{\prime}\right)^{2}+\left(y-y^{\prime}\right)^{2}}} d l^{\prime}$

This is an integral equation where $\sigma(x, y)$ must be determined in order to compute the potential $\varphi(x, y) . K$ is an arbitrary constant which represents the distance to the reference point where the potential is zero. This constant must be included so (8) can have a proper solution. For high values of $K$ every point of the domain $R$ can be treated as being at the same distance from the reference point with minimal influence on the final 
results. In our tests we have set $K>500$. By using pulse functions, the flux sources of (8) can be approximated with piecewise step functions. To achieve this, first the contour $C$ must be discretised into line segments. A simple way of doing this is to define $N$ points. Each of these $\Delta C_{i}$ line segments are described by two neighbouring points $\left(x_{i}, y_{i}\right)$ and $\left(x_{i+1}, y_{i+1}\right)$ for $i=$ $1,2, \ldots N$. Now we can approximate the flux sources with pulse functions as follows:

$\sigma \cong \sum_{i=1}^{N} \sigma_{i} P_{i}(l)$

where $P_{i}(l)$ is a pulse function which has a value of 1 on $\Delta C_{i}$ and 0 elsewhere. If we substitute (9) in (8) we obtain the approximation for the integral equation

$\varphi(x, y) \cong \sum_{i=1}^{N} \sigma_{i} \psi_{i}(x, y)$

where

$\psi_{i}(x, y)=\int_{\Delta C_{i}} \ln \frac{K}{\sqrt{\left(x-x^{\prime}\right)^{2}+\left(y-y^{\prime}\right)^{2}}} d l^{\prime}$

Next, we need to define midpoints of each of the $\Delta C_{j}$ segments denoted with $\widehat{r}_{j}=\left(x_{j}, y_{j}\right)$ for $j=1,2, \ldots N$. Substituting (10) in (1) we get

$\sum_{i=1}^{N} \sigma_{i} l_{j i}=\gamma_{j}$

where $\gamma_{j}=\gamma\left(\widehat{r}_{j}\right)$ and

$l_{j i}=\left[\alpha \psi_{i}\right]_{(x, y)=\widehat{r}_{J}}$

for Dirichlet boundary conditions. Solving for $\sigma$, the potential $\varphi$ in matrix notation has the final form

$\varphi=[\psi][\sigma]=[\psi][l]^{-1}[\gamma]$

Every term of $[\psi]$ has an analytical solution based on (11) for an arbitrary point $(x, y)$ in $R$. The square matrix $[l]$ holds the characteristics of the approximated system. From (14) we can conclude that the potential $\varphi$ can be calculated for any arbitrary point $(x, y)$. This is possible because of the superposition expression in (8) and the discretisation of $C$ only, which allows calculating $\varphi$ at points in a tightly packed grid or at only one point of interest.

\subsection{Analytical solutions}

In order to verify the methods previously laid out, exact analytical solutions for the Laplace's equation are needed. The solution of a boundary problem in Cartesian coordinate system requires finding the solution of the partial differential equation for which the boundary conditions are met. By applying the method of separation of variables, the partial differential equations can be reduced to ordinary ones [6]. Many analytical expressions can be obtained in this way and are provided in textbooks. The values of the numerical solution converge to the ones of the analytical solution. When comparing results, the discretization factor should be considered. Some boundary values are approximated after discretization in some cases and will have an impact on its surrounding points. To reduce this effect we avoid analysing points near the boundaries.

\section{Results and discussions}

In this section we present comparison of results obtained by implementing the FDM, FEM and MoM numerical methods. We will compare the accuracy of the numerical solutions with the exact analytical solution for different levels of domain discretization of a problem.

We shall consider a rectangular domain as shown in Figure 1. The domain is placed in the first quadrant of the coordinate system. The value $p$ represents the length and the value $q$ the width of the domain. For simplicity we divide the domain in $N \times M$ elements where $N$ is the number of elements on $\mathrm{x}$ axis and $M$ the number of elements on y axis. Next, we obtain the spacing between each element needed for (3) and (5) as $\Delta x=a={ }^{p} / N$ and $\Delta y=b=q / M$. At each coordinate of the formed grid we calculate the electric potential. Because MoM requires only the boundary be discretised in total of $N \cdot M$ segments, we will calculate the potential at the same points as the other methods.

It should be noted that the coordinates of the nodes used in FDM do not match with the coordinates of the elements in FEM when using the same discretization level. To overcome this situation the calculated values at each node in FDM are bilinearly interpolated to correspond with the coordinates of FEM.

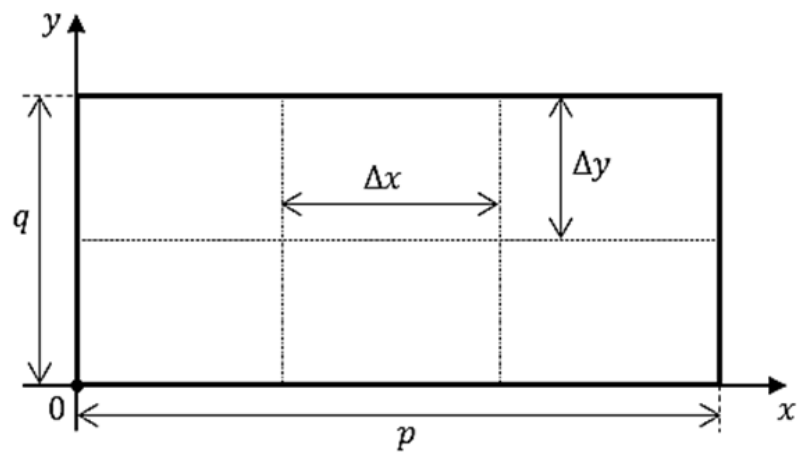

Figure 1. Considered test domain for numerical calculation.

For our first test we will set the length of the domain as $p \gg q$. This allows the whole problem to be treated as $1 \mathrm{D}$ with known exact solution at the middle points of the $\mathrm{x}$ axis. The length of the domain will be set to 1 and the width to 0.01 . If we consider the boundary problem where the bottom, left and right boundaries are set at potential $0 \mathrm{~V}$, and the potential of the top boundary $\varphi(x, q)=10 \mathrm{~V}$, the exact $1 \mathrm{D}$ solution at $x=0.5$ is

$\varphi(y)=\frac{10}{0.01} y, \quad 0<y<q$

Table 1 shows the results of the 1D approximation with various discretization levels at $y=0.875$. The exact solution at this point is $8.75 \mathrm{~V}$. Because of the symmetry in the problem MoM converges to the exact solution with low level of discretization. Calculations based on superposition have a big impact in the end results of symmetrical problems. For this 1D approximation the effects of the side boundaries are annulled at the middle points and the linear change of the potential on the $y$ axis makes MoM very suitable for these types of problems. The case for FEM is opposite of MoM because the 
elements of the domain are stretched out so a denser mesh is required to achieve good accuracy. The numerical solution for FDM was achieved with a fixed minimal difference value of $10^{-5}$ as a limit factor for the maximum number of iterations. For a low number of nodes, the numerical solution converges to the exact value with good accuracy and low number of iterations. However, increasing the node density did not yield any significant improvements in the results, even when the minimal difference value was lowered to $10^{-10}$. This is expected because the term $\Delta x / \Delta y$ in (3) does not have a value close to 1 and increases the error in this computation. To overcome this issue a better grid should be considered when dealing with problems of this type.

Table 1. Calculated potential for the $1 \mathrm{D}$ case at $\boldsymbol{y}=\mathbf{0 . 8 7 5}$ for 3 different discretization levels.

\begin{tabular}{cccc}
\hline $\boldsymbol{N} \times \boldsymbol{M}$ & $\boldsymbol{\varphi}_{\mathrm{FDM}}, \boldsymbol{V}$ & $\boldsymbol{\varphi}_{\mathrm{FEM}}, \boldsymbol{V}$ & $\boldsymbol{\varphi}_{\mathrm{MoM}}, \boldsymbol{V}$ \\
\hline $\mathbf{3} \times \mathbf{4}$ & 8.7497492 & 9.1224472 & 8.7500820 \\
$\mathbf{9} \times \mathbf{1 2}$ & 8.7499462 & 8.7338693 & 8.7500001 \\
$\mathbf{2 7} \times \mathbf{3 6}$ & 8.7494397 & 8.7500001 & 8.7500158 \\
\hline
\end{tabular}

Next, we shall consider a domain where $p=q=4$. For this 2D problem the boundary potentials on the sides and bottom will remain at $0 \mathrm{~V}$. The potential at the top boundary will have the form

$\varphi(x, q)=\frac{1}{2} \sin \left(\frac{\pi x}{4}\right), \quad 0<x<4$

The exact solution in the domain is given by

$\varphi(x, y)=\frac{1}{2 \sinh \pi} \sin \left(\frac{\pi x}{4}\right) \sinh \left(\frac{\pi y}{4}\right)$

We compute the numerical solutions at 3 arbitrary points, away from the boundaries, with each method for different levels of discretization. The numerical solutions are then compared with the exact solution. The points of interest are $P_{1}(1,3), P_{2}(2.2,3)$ and $P_{3}(2.2,1.8)$. When computing the numerical solution for different discretization, not all methods have an element with a corresponding coordinate at each point of interest. Because of this, all values at the missing coordinates are calculated based on the 4 closest elements by bilinear interpolation.

In Tables 2-5 the obtained results of the computations at points $P_{1}, P_{2}, P_{3}$ are presented. FEM has the greatest accuracy in the numerical solutions for the different discretization levels overall. The accuracy of MoM depends directly on the segmented boundary and its values. For the piecewise step approximation, each linear segment receives an average of the boundary values at its end points. This in turn increases the error of the numerical solution when continuous functions are used for the boundaries. As a general rule, a denser grid means greater accuracy. However, this is not always true for FDM. The results shown of the FDM solution were obtained for a fixed minimal difference value of $10^{-5}$. It was observed that a denser grid would mean slower convergence rate. This means that small changes in value per iteration can result in premature end of the iteration process. In this case a high number of iterations are required for an increase in accuracy.

Table 2. Numerical solutions at points $\boldsymbol{P}_{1}, \boldsymbol{P}_{2}, \boldsymbol{P}_{\mathbf{3}}$ for $\mathbf{1 0} \times \mathbf{1 0}$ discretization.

\begin{tabular}{ccccc} 
Solution & Exact & FDM & FEM & MoM \\
\cline { 2 - 5 } $\boldsymbol{\varphi ( \boldsymbol { P } _ { 1 } ) , \boldsymbol { V }}$ & 0.1600493 & 0.1608919 & 0.1590626 & 0.1578575 \\
$\boldsymbol{\varphi}\left(\boldsymbol{P}_{\mathbf{2}}\right), \boldsymbol{V}$ & 0.2235572 & 0.2247342 & 0.2221789 & 0.2206091 \\
$\boldsymbol{\varphi}\left(\boldsymbol{P}_{\mathbf{3}}\right), \boldsymbol{V}$ & 0.0827006 & 0.0835499 & 0.0816390 & 0.0815849 \\
\hline
\end{tabular}

Table 3. Numerical solutions at points $\boldsymbol{P}_{1}, \boldsymbol{P}_{2}, \boldsymbol{P}_{3}$ for $20 \times 20$ discretization.

\begin{tabular}{lcccc}
\hline Solution & Exact & FDM & FEM & MoM \\
\cline { 2 - 5 } $\boldsymbol{\varphi}\left(\boldsymbol{P}_{\mathbf{1}}\right), \boldsymbol{V}$ & 0.1600493 & 0.1598963 & 0.1598024 & 0.1595207 \\
$\boldsymbol{\varphi}\left(\boldsymbol{P}_{\mathbf{2}}\right), \boldsymbol{V}$ & 0.2235572 & 0.2233435 & 0.2232124 & 0.2228383 \\
$\boldsymbol{\varphi}\left(\boldsymbol{P}_{\mathbf{3}}\right), \boldsymbol{V}$ & 0.0827006 & 0.0821843 & 0.0824355 & 0.0824305 \\
\hline
\end{tabular}

Table 4. Numerical solutions at points $\boldsymbol{P}_{1}, \boldsymbol{P}_{2}, \boldsymbol{P}_{3}$ for $\mathbf{3 0} \times \mathbf{3 0}$ discretization.

\begin{tabular}{lcccc}
\hline Solution & Exact & FDM & FEM & MoM \\
\cline { 2 - 5 } $\boldsymbol{\varphi ( P _ { 1 } ) , \boldsymbol { V }}$ & 0.1600493 & 0.1592563 & 0.1599389 & 0.1598184 \\
$\boldsymbol{\varphi}\left(\boldsymbol{P}_{\mathbf{2}}\right), \boldsymbol{V}$ & 0.2235572 & 0.2224496 & 0.2234031 & 0.2232416 \\
$\boldsymbol{\varphi}\left(\boldsymbol{P}_{\mathbf{3}}\right), \boldsymbol{V}$ & 0.0827006 & 0.0810601 & 0.0825825 & 0.0825824 \\
\hline
\end{tabular}

Table 5. Numerical solutions at points $\boldsymbol{P}_{1}, \boldsymbol{P}_{2}, \boldsymbol{P}_{\mathbf{3}}$ for $\mathbf{4 0} \times \mathbf{4 0}$ discretization.

\begin{tabular}{|c|c|c|c|c|}
\hline Solution & Exact & FDM & FEM & MoM \\
\hline$\varphi\left(P_{1}\right), V$ & 0.1600493 & 0.1584903 & 0.1599873 & 0.1599205 \\
\hline$\varphi\left(P_{2}\right), V$ & 0.2235572 & 0.2213794 & 0.2234706 & 0.2233806 \\
\hline$\varphi\left(P_{3}\right), V$ & 0.0827006 & 0.0796165 & 0.0826342 & 0.0826345 \\
\hline
\end{tabular}

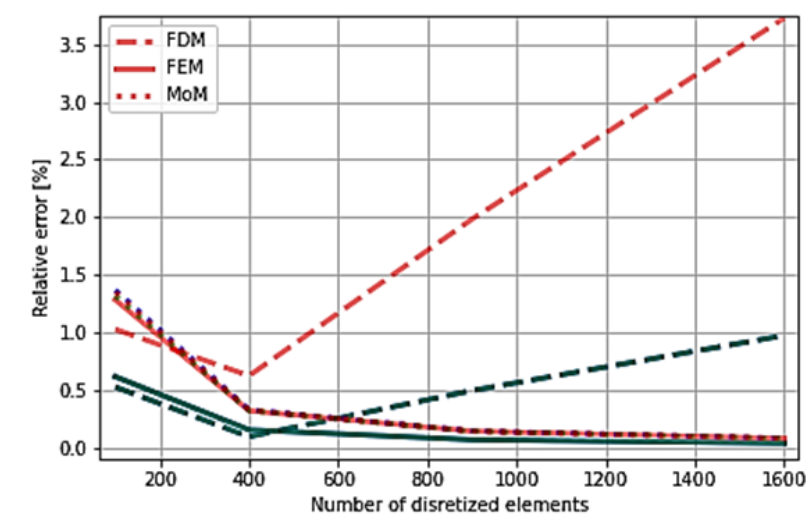

Figure 2. Relative error of the numerical solution for each method. Red lines show values at $\boldsymbol{P}_{\mathbf{1}}$, green at $\boldsymbol{P}_{\mathbf{2}}$, blue at $\boldsymbol{P}_{\mathbf{3}}$.

The relative error for each method is shown in Figure 2. The dashed lines represent the error of FDM, the solid lines FEM and the dotted lines MoM for different discretization levels. The results show that FDM has the highest relative error and is directly connected to the convergence rate. This means that denser grid requires a higher number of iterations to maintain the same accuracy. While FEM has the lowest error overall, at the 
points near the center of the domain the error rises. This comes from the basis of the method where the numerical solution must satisfy the boundary conditions at a cost of reduced differential equations. The error of MoM is more consistent for better discretization because the numerical solution is formulated to converge to the exact solution while the boundary conditions do not have to be fully met.

Lastly, we compare the computational time needed for each method to achieve the same average accuracy level for an appropriately discretised domain. The target average absolute error was set to $\sim 10^{-5}$. The solution with MoM was calculated on a grid formed by its level of discretization $N \times M$. The measured times are shown in Table 6. FEM achieves the target accuracy with the least amount of time. This is due to the lower discretization level. With MoM it takes a longer time to reach the target mostly because of the number of linear segments needed for this problem. If a different problem with constant boundary values was considered, the targeted accuracy would be achieved with less amount of time and segments. Based on the implementations of the methods in the previous section, for higher discretization level the time ratio of a solution based on FEM or MoM can be completely different. This is because the operations in FEM mainly consist of memory and data manipulations, whereas in MoM mainly consist of mathematical calculations from multiple sources. The case for FDM is different because of its iterative approach in achieving convergence where higher number of iterations mean better accuracy.

Table 6. Time measured for reaching the required average accuracy of the numerical solutions.

\begin{tabular}{cccc}
\hline Solution & FDM & FEM & MoM \\
\cline { 2 - 4 } $\boldsymbol{N} \times \boldsymbol{M}$ & $80 \times 80$ & $70 \times 70$ & $100 \times 100$ \\
$\boldsymbol{t}, \boldsymbol{s}$ & 250 & 44 & 59 \\
\hline
\end{tabular}

\section{Conclusion}

In this paper we have presented our implementations of the FDM, FEM and MoM numerical methods and investigated their accuracy on a rectangular domain with
Dirichlet boundary conditions. All the calculations and implementations of the numerical solutions were written in the free programming language Python. For problems where lower accuracy is acceptable, FDM can be used with the lowest computational cost. MoM has greater accuracy than FDM and shows great results even with very low number of segments. Because of its flexibility and accuracy, FEM can be used for solving almost any type of boundary problem. In cases where high accuracy and dense mesh is required, FEM has high memory demands and will increase the computational time exponentially. The computational requirements of MoM are lower in comparison due to its superposition approach and the ability to calculate only at points of interest. When dealing with problems with high symmetry different discretization types can produce different results near the boundary of the domain. This is especially true for FEM because its formulation must satisfy the boundary conditions, while MoM strives for better accuracy in the middle of the domain in comparison.

\section{References}

[1] Sadiku, M. N. O. \& Peterson, A. F. (1990). A comparison of numerical methods for computing electromagnetic fields, IEEE Proceedings on Southeastcon, vol. 1, pp. 42-47, doi: 10.1109/SECON.1990.117766.

[2] Sarkar, T., Chung, Y. \& Palma, M. (2002). Solution of the General Helmholtz Equation Starting from Laplace's Equation. Annual Review of Progress in Applied Computational Electromagnetics. vol. 17, no. 3.

[3] Smith, G. D. (1985). Numerical Solution of Partial Differential Equations: Finite Difference Methods. Third Edition, Oxford Applied Mathematics and Computing Science Series, pp. 6-9.

[4] Shabbir, M., Malik, M., Ahmad, M., Pervaiz, A. \& Siddique, R. (2012). Finite Element Solution for Two Dimensional Laplace Equation tablewith Dirichlet Boundary Conditions, Pak. J. Engg. \& Appl. Sci., vol. 10, pp. 97-102. [5] Harrington, R. F., Pontoppidan, K., Abrahamsen P., \& Abertsen, N. C. (1969). Computation of Laplacian potentials by an equivalent source method, Proc. IEE, vol. 116, no. 10, pp. $1715-1920$

[6] Bland, D. R. (1961). Solutions of Laplace's Equations, pp. 15-28, https://doi.org/10.1007/978-94-011-7694-1. 